\title{
SOME SOLUTIONS OF A NONLINEAR DIFFERENTIAL EQUATION OF HIGH ORDER*
}

\author{
BY \\ P. E. W. GRENSTED \\ University of Cambridge
}

\begin{abstract}
Some exact monotonic, and approximate oscillatory, solutions of the nonlinear equation $d^{n} y / d x^{n}=K|y|^{r} \operatorname{sgn} y, 0 \leq r$, are derived. The coefficient $K$ may be positive or negative, $r$ may be non-integral and $n$ is any positive integer. For the case $r=0$, exact solutions in closed form are obtained. The conditions under which the approximate solutions will be highly accurate are discussed. Every component of the general solution of the linear equation $d^{n} y / d x^{n}=K y$ is shown to be analogous to a corresponding solution of the given nonlinear equation.
\end{abstract}

1. Introduction of equation. Equations of the type

$$
\frac{d^{n} y}{d x^{n}}=K|y|^{r} \operatorname{sgn} y, \quad 0 \leq r \neq 1,
$$

sometimes occur in applied mechanics. Two examples† are quoted here: If $n=4$ and $K<0$, equation (1) governs the bending moment $y$ at a distance $x$ along a uniform beam embedded in a linear elastic medium. The material of the beam is such that the curvature of the beam is proportional to the $r$-th power of the bending moment. Conversely, if $y$ represents the transverse deflection of the beam, the equation applies to a linear beam embedded in an elastic medium whose local deflection is proportional to the $(1 / r)$-th power of the local pressure.

The second example is taken from a problem in the theory of optimal control. A plant, with transfer function $1 / \mathrm{s}^{\mathrm{m}}$, has output $v(t)$ and a control input $u(t)$ which is subject to the saturation constraint $|u| \leq 1$. The governing equation of the plant is therefore

$$
\frac{d^{m} v}{d t^{m}}=u(t)
$$

The desired output of the plant is zero. For any given initial state of the plant at $t=0$, it is required to find the $u(t)$, and the corresponding trajectory of the plant state, that will minimize the integral of squared error, namely $\int_{0}^{\infty} v^{2} d t$. It can be shown [1], by application of Pontryagin's maximum principle, that the required trajectory is given by the solution of

$$
\frac{d^{2 m} y}{d t^{2 m}}=-(-1)^{m} \operatorname{sgn} y,
$$

where $v=d^{m} y / d t^{m}$, with the boundary conditions that $y$ and its first $2 m-1$ derivatives become zero at $t=+\infty$, and that $v$ and its derivatives correspond to the given state of the plant at $t=0$. Equation (2) is an example of (1) in which $r=0$.

*Received February 24, 1965; revised manuscript received December 13, 1965.

$\dagger$ Due to F. A. Leckie and A. T. Fuller respectively. Private discussion. 
An explicit general solution of (1) is not known, but certain special exact and approximate solutions can be derived. These solutions have the property that $y(x)$ either converges to or diverges from zero as $x$ increases, or is periodic. It will be shown that these special solutions of (1) are analogous to each of the modes of the solution of the linear equation

$$
\frac{d^{n} y}{d x^{n}}=K y
$$

The solution of (3) may be written

$$
y=\sum A_{k} e^{\rho_{k} x},
$$

where the $\rho_{k}(k=1,2, \cdots n)$ are the $n$ roots of $\rho^{n}=K$. If $\rho_{k}$ is real, the corresponding term in (4) is divergent monotonic or convergent monotonic if $\rho_{k}>0$ or $\rho_{k}<0$ respectively. If $\rho_{k}$ is complex, the two terms in (4) corresponding to $\rho_{k}$ and $\bar{\rho}_{k}$ combined into a single term which is divergent oscillatory, periodic or convergent oscillatory if $\operatorname{Re} \rho_{k}>0$, $\operatorname{Re} \rho_{k}=0$ or $\operatorname{Re} \rho_{k}<0$ respectively. By considering the location of the roots of $\rho^{n}=K$ in the complex plane, it can be shown that, for given $n$ and sign of $K$, the number of modes of each of the five types which make up the solution of (3) are as set out in Table 1.

The object of the following is to derive solutions of (1) corresponding to each mode of the solution of (3), and having the same properties, viz convergent, divergent or periodic, and oscillatory with two arbitrary constants or monotonic with one arbitrary constant. Solutions of these types are already known [1] for the particular case $n=6$, $r=0$ and $K>0$.

In the following three sections, monotonic, periodic, and oscillatory solutions of (1)

TABLE 1

\begin{tabular}{|c|c|c|c|c|c|c|c|}
\hline & & & \multicolumn{5}{|c|}{ number of distinct modes } \\
\hline \multirow{2}{*}{\multicolumn{3}{|c|}{$(p=0,1,2, \cdots)$}} & \multicolumn{2}{|c|}{$\begin{array}{l}\text { monotonic, with one } \\
\text { arbitrary constant }\end{array}$} & \multicolumn{3}{|c|}{$\begin{array}{l}\text { oscillatory, with two } \\
\text { arbitrary constants }\end{array}$} \\
\hline & & & convergent & divergent & convergent & periodic & divergent \\
\hline$K>0$ & \multicolumn{2}{|c|}{$\begin{array}{l}4 p+1 \\
4 p+2 \\
4 p+3 \\
4 p+4\end{array}$} & $\begin{array}{l}0 \\
1 \\
0 \\
1\end{array}$ & $\begin{array}{l}1 \\
1 \\
1 \\
1\end{array}$ & $\begin{array}{c}p \\
p \\
p+1 \\
p\end{array}$ & $\begin{array}{l}0 \\
0 \\
0 \\
1\end{array}$ & $\begin{array}{l}p \\
p \\
p \\
p\end{array}$ \\
\hline$K<0$ & \multicolumn{2}{|c|}{$\begin{array}{l}4 p+1 \\
4 p+2 \\
4 p+3 \\
4 p+4\end{array}$} & $\begin{array}{l}1 \\
0 \\
1 \\
0\end{array}$ & $\begin{array}{l}0 \\
0 \\
0 \\
0\end{array}$ & $\begin{array}{c}p \\
p \\
p \\
p+1\end{array}$ & $\begin{array}{l}0 \\
1 \\
0 \\
0\end{array}$ & $\begin{array}{c}p \\
p \\
p+1 \\
p+1\end{array}$ \\
\hline \multicolumn{2}{|c|}{$\begin{array}{l}\text { Corresponding solu- } \\
\text { tions of equation (1) }\end{array}$} & $\begin{array}{l}r>1 \\
r<1\end{array}$ & $\begin{array}{l}(15) \\
(11)\end{array}$ & $\begin{array}{l}(11) \\
(15)\end{array}$ & $\begin{array}{l}(33) \\
(35)\end{array}$ & $\begin{array}{l}(22) \\
(22)\end{array}$ & $\begin{array}{l}(35) \\
(33)\end{array}$ \\
\hline
\end{tabular}

Type and number of the modes of the solution of $\frac{d^{n} y}{d x^{n}}=K y$ 
are derived. Their form depends on a parameter $q$, where

$$
q=\frac{n}{1-r}, \quad 0 \leq r \neq 1 .
$$

It may be noted that

$$
q \geq n \quad \text { if } \quad r<1 ; \quad q<0 \text { if } r>1 .
$$

2. Monotonic solutions. Substitution of

$$
y=A\left(x_{0}-x\right)^{\natural}, \quad x<x_{0},
$$

into (1) yields

$(-)^{n} A q(q-1)(q-2) \cdots(q-n+1)\left(x_{0}-x\right)^{q-n}=K|A|^{r}\left(x_{0}-x\right)^{q r} \operatorname{sgn} A$.

Equation (8) is satisfied identically only if $q$ is given by (5) and

$$
A= \pm\left[(-)^{n} q(q-1) \cdots(q-n+1) / K\right]^{1 /(r-1)},
$$

with the expression in square brackets positive. Using (6), it follows that (8) can be satisfied only if

$$
\left.\begin{array}{ll}
\text { either } \quad K>0, n \text { even, } \\
\text { or } \quad K>0, n \text { odd, } \quad r>1, \\
\text { or } \quad K<0, n \text { odd, } \quad r<1 .
\end{array}\right\}
$$

Hence, provided one of conditions (10) holds, (1) has solution

$$
y=A\left(x_{0}-x\right)^{n /(1-r)}, \quad x<x_{0},
$$

where $x_{0}$ is arbitrary and $A$, which is of arbitrary sign, is given by (9) and (5). Solution (11) is divergent monotonic if $r>1$, and is convergent monotonic if $r<1$.

Similarly

$$
y=A\left(x-x_{0}\right)^{a}, \quad x>x_{0},
$$

satisfies (1) only if $q$ is given by (5) and

$$
A= \pm[q(q-1) \cdots(q-n+1) / K]^{1 /(r-1)},
$$

with the expression in square brackets positive; i.e.

$$
\left.\begin{array}{ll}
\text { either } \quad K>0, n \text { even, } \\
\text { or } \quad K>0, n \text { odd, } \quad r<1, \\
\text { or } \quad K<0, n \text { odd, } \quad r>1 .
\end{array}\right\}
$$

Hence, provided one of conditions (14) holds, (1) has solution

$$
y=A\left(x-x_{0}\right)^{n /(1-r)}, \quad x>x_{0},
$$

where $x_{0}$ is arbitrary and $A$, which is of arbitrary sign, is given by (13) with (5). Solution (15) is divergent monotonic if $r<1$, and is convergent monotonic if $r>1$. 
Solutions (11) and (15) of (1) correspond to all the monotonic modes listed in Table 1.

3. Periodic solutions. Periodic solutions of (1) may be found approximately by the method of equivalent linearization [2]. In the present application this method becomes equivalent to Galerkin's method, to the describing function method and to the method of harmonic balance [2]. Here, we assume a periodic solution of the form

$$
y=A \sin (\gamma x+\phi), \quad A>0,
$$

and replace the right hand side of (1) by a linear term $K^{\prime} y$. The coefficient $K^{\prime}$ is chosen to minimize the mean square error of the right hand side of (1). Thus

$$
\frac{\partial}{\partial K^{\prime}} \int_{0}^{2 \pi / \gamma}\left(K|y|^{r} \operatorname{sgn} y-K^{\prime} y\right)^{2} d x=0,
$$

where $y$ is of the assumed form (16).

From (17) we obtain

$$
K^{\prime}=A^{r-1} c_{r} K,
$$

where

$$
c_{r}=\frac{4}{\pi} \int_{0}^{\pi / 2} \sin ^{r+1} u d u=\frac{2 \Gamma\left(\frac{r+2}{2}\right)}{\pi^{\frac{1}{2}} \Gamma\left(\frac{r+3}{2}\right)} .
$$

Result (18) is in agreement with the describing function given by Greif [3]. Values of $c_{r}$ for $0 \leq r \leq 2$ are given in Table 2 . The table can be extended by using the recurrence relation

$$
c_{r+2}=\frac{r+2}{r+3} c_{r},
$$

which follows from (18).

The approximate form of (1) is

$$
\frac{d^{n} y}{d x^{n}}=A^{r-1} c_{r} K y .
$$

The assumed form (16) of $y$ will satisfy (20) if, and only if,

$$
\left.\begin{array}{ll}
\text { either } & n=4 p+4, \quad K>0, \\
\text { or } & n=4 p+2, \quad K<0 .
\end{array}\right\} \quad(p=0,1,2, \cdots)
$$

TABLE 2

Values of $c_{r}$ defined by (18)

\begin{tabular}{cccccccc}
\hline$r$ & 0 & $1 / 4$ & $1 / 3$ & $1 / 2$ & $2 / 3$ & $3 / 4$ & 1 \\
$c_{r}$ & 1.27324 & 1.18523 & 1.15960 & 1.11284 & 1.07119 & 1.05201 & 1.00000 \\
\hline$r$ & $5 / 4$ & $4 / 3$ & $3 / 2$ & $5 / 3$ & $7 / 4$ & 2 & $r>2$ \\
$c_{r}$ & 0.95490 & 0.94115 & 0.91531 & 0.89147 & 0.88021 & 0.84883 & $\frac{r}{r+1} c_{r-2}$
\end{tabular}


If either of conditions (21) holds, a solution of (1) is approximately

$$
y=A \sin (\gamma x+\phi),
$$

where $A(A>0)$ and $\phi$ are arbitrary, and

$$
\gamma=\left|K c_{r} A^{r-1}\right|^{1 / n} \text {. }
$$

Approximate solution (22) of (1) corresponds to all the periodic modes listed in Table 1.

4. Convergent and divergent oscillatory solutions. Let

$$
y=\left(x-x_{0}\right)^{n /(1-r)} \eta, \quad x>x_{0},
$$

and

$$
x-x_{0}=e^{\theta} .
$$

On applying these changes of variable, (1) becomes

$$
\left(\frac{d}{d \theta}+q\right)\left(\frac{d}{d \theta}+q-1\right) \ldots\left(\frac{d}{d \theta}+q-n+1\right) \eta=K|\eta|^{r} \operatorname{sgn} \eta,
$$

where $q$ is defined by (5).

Periodic solutions of (26), of the approximate form

$$
\eta(\theta)=A \sin (\beta \theta+\phi), \quad A>0,
$$

may be found by the method of equivalent linearization. Following the procedure used to find periodic solutions of (1), the right hand side of (26) is replaced by $A^{r-1} c_{r} K \eta$. The resulting linear equation is satisfied by (27) if, and only if,

$$
(j \beta+q)(j \beta+q-1) \cdots(j \beta+q-n+1)=A^{r-1} c_{r} K .
$$

Values $\beta_{m}\left(\beta_{m}>0\right)$ of $\beta$ which satisfy (28) must be such that the left hand side of (28) is real, and of the same sign as $K$. Since the angle of each factor on the left hand side of (28) lies between 0 and $\pi / 2$ if $r<1$, and between $\pi / 2$ and $\pi$ if $r>1$, this condition may be expressed as follows. If

$$
\left.\begin{array}{ll}
\text { either } & K>0, n \text { even, } \\
\text { or } & K>0, n \text { odd, } \quad r<1, \\
\text { or } & K<0, n \text { odd, } \quad r>1,
\end{array}\right\}
$$

then

$$
\sum_{s=0}^{n-1} \tan ^{-1}\left|\frac{\beta_{m}}{q-s}\right|=2 m \pi \quad(m=1,2, \cdots M) .
$$

If

$$
\left.\begin{array}{ll}
\text { either } & K<0, n \text { even, } \\
\text { or } & K<0, n \text { odd, } \quad r<1, \\
\text { or } & K>0, n \text { odd, } \quad r>1,
\end{array}\right\}
$$


then

$$
\sum_{i=0}^{n-1} \tan ^{-1}\left|\frac{\beta_{m}}{q-s}\right|=(2 m-1) \pi \quad(m=1,2, \cdots M) .
$$

Each of the $n$ terms of the sums in (30) and (32) can contribute an angle of between 0 and $\pi / 2$. Hence the total number $M$ of distinct values $\beta_{m}\left(\beta_{m}>0\right)$ satisfying (30) or (32) is the greatest integer less than $n / 4$ or $(n+2) / 4$ respectively. All the solutions of (30) and (32) are shown in Fig. 1 for $n \leq 8,0 \leq r \leq 5$.

Approximate oscillatory solutions of (1) are thus, from (27), (25) and (24),

$y=\left(x-x_{0}\right)^{n /(1-r)} A_{m} \sin \left[\beta_{m} \log \left(x-x_{0}\right)+\phi\right], \quad x>x_{0}, \quad(m=1,2, \cdots M)$

where $x_{0}$ and $\phi$ are arbitrary constants, $\beta_{m}\left(\beta_{m}>0\right)$ is a root of (30) if one of conditions (29) holds or a root of (32) if one of conditions (31) holds, and, from (28),

$$
A_{m}=\left|\left(j \beta_{m}+q\right)\left(j \beta_{m}+q-1\right) \cdots\left(j \beta_{m}+q-n+1\right) /\left(K c_{r}\right)\right|^{1 /(r-1)}
$$

where $q=n /(1-r)$.

It is easy to verify that the number $M$ of approximate solutions (33) corresponds to the number of divergent oscillatory modes listed in Table 1 if $r<1$, and to the number of convergent oscillatory modes listed in Table 1 if $r>1$.

A further set of oscillatory solutions of (1) can be derived by noting that (1) is unchanged if $x$ is replaced by $2 x_{0}-x$ and $K$ by $(-)^{n} K$. Making these changes in (33) and conditions (29) and (31) we obtain approximate solutions of (1):

$y=\left(x_{0}-x\right)^{n /(1-r)} A_{m} \sin \left[\beta_{m} \log \left(x_{0}-x\right)+\phi\right], \quad x<x_{0}, \quad(m=1,2, \cdots M)$

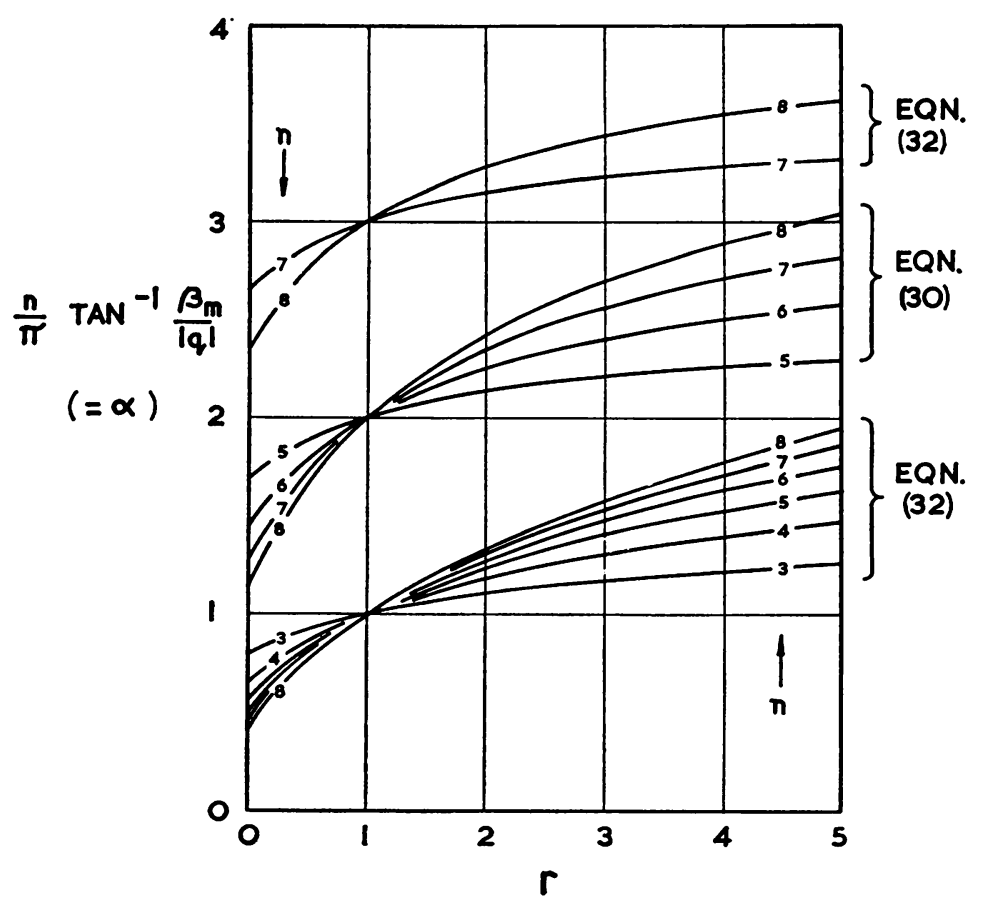

Fig. 1. All solutions of (30) and (32) for $0 \leq r \leq 5$ and $n \leq 8$. $\beta_{m}=|q| \tan (\pi \alpha / n)$, where $\alpha$ is ordinate. 
where $x_{0}$ and $\phi$ are arbitrary constants, $A_{m}$ is given by (34) and $\beta_{m}$ is given by (30) if

$$
\left.\begin{array}{ll}
\text { either } \quad K>0, n \text { even, } \\
\text { or } \quad K<0, n \text { odd, } \quad r<1, \\
\text { or } \quad K>0, n \text { odd, } \quad r>1
\end{array}\right\}
$$

alternatively $\beta_{m}$ is given by (32) if

$$
\left.\begin{array}{ll}
\text { either } & K<0, n \text { even, } \\
\text { or } & K>0, n \text { odd, } \quad r<1, \\
\text { or } & K<0, n \text { odd, } \quad r>1 .
\end{array}\right\}
$$

It is easily verified that the number $M$ of approximate solutions (35) corresponds to the number of convergent oscillatory modes listed in Table 1 if $r<1$, and to the number of divergent oscillatory modes listed in Table 1 if $r>1$. Thus the solutions (33) and (35) correspond to all the non-periodic oscillatory modes listed in Table 1.

5. Accuracy of periodic solutions. We now consider the accuracy of the periodic solution (22) of (1). If (22) is regarded as a first approximation $y_{1}$ to the true solution of (1), we have

$$
y_{1}=A \sin (\gamma x+\phi),
$$

where $A$ is related to $\gamma$ by (23).

An improved approximation $y_{1}^{*}$ can be obtained by substituting $y_{1}$ into the right hand side of (1):

$$
\frac{d^{n} y_{1}^{*}}{d x^{n}}=K\left|y_{1}\right|^{r} \operatorname{sgn} y_{1} .
$$

On expanding the right hand side of (39) as a Fourier series, we obtain

$$
\frac{d^{n} y_{1}^{*}}{d x^{n}}=K A^{n} c_{r}\left[\sin (\gamma x+\phi)+\sum_{s=3,5,7, \ldots} h_{r, s} \sin s(\gamma x+\phi)\right],
$$

where

$$
c_{r} h_{r, 8}=\frac{4}{\pi} \int_{0}^{\pi / 2}(\sin u)^{r} \sin s u d u
$$

and $c_{r}$ is given by (18). It can be shown from (41) that $h_{r, s}$ satisfies the recurrence relation

$$
h_{r, s+2}=\frac{s-r}{s+r+2} h_{r, s}, \quad s \text { odd, }
$$

with $h_{r, 1}=1$.

Since $y_{1}^{*}$ is periodic and $n$ is even, we obtain by integrating (40) and using (23)

$$
y_{1}^{*}=A\left[\sin (\gamma x+\phi)+\sum_{s=3,5,7, \ldots} \frac{h_{r, s}}{s^{n}} \sin s(\gamma x+\phi)\right] .
$$

The improved approximation (43) is equal to the first approximation plus harmonic 
terms. The relative magnitude of these harmonic terms is a measure of the error with which the approximate solution (38) satisfied the original equation (1). This relative magnitude can be measured as $e$, where $e^{2}$ is the ratio of the mean square of the harmonic terms to the mean square of the fundamental term in (43). Thus

$$
e^{2}=\sum_{s=3,5,7, \ldots}\left(\frac{h_{r, s}}{s^{n}}\right)^{2} .
$$

We now assume (without proof) that, if $e$ is very small, the iteration procedure used above, if repeated, would rapidly converge to the exact solution of (1), and that $e$ is then also a measure of the error between (38) and the exact solution of (1). For given $r$, $e$ decreases rapidly as $n$ increases. For instance, if $n=2$, and $r=0$ or $r=3$, then $e \bumpeq$ $1 / 27$; if $n=4$, and $r=0$, or $r=3$, then $e \bumpeq 1 / 243$. For $0 \leq r \leq 3$, these values of $e$ suggest that frequency $\gamma$ will be related to amplitude $A$ by (23) to within about $3 \%$ if $n=2$ and to better than $1 \%$ if $n \geq 4$.

This conclusion is readily confirmed for $n=2$ by a numerical comparison of (23) with the exact relation

$$
\gamma=\frac{2[2(1+r)]^{1 / 2}}{(3+r) c_{2 /(1+r)}}\left(-K A^{r-1}\right)^{1 / 2}
$$

Equation (45) has been derived from the exact relation between period and amplitude given, for instance, by Kauderer [4]. Moreover, for $0<r \leq 3$ at least, and $n=2$, there is also excellent numerical agreement between (43) (with (23)) and the exact periodic solution of (1) given by Rosenberg [5] in terms of incomplete Beta functions.

If derivatives of $y$ are required to the same accuracy as $y_{1}$, solution (43) should be used.

Exact periodic solutions when $r=0$. If $r=0$, solution (43) with (23) is an exact solution of (1), for in this case the right hand side of (39) is unchanged if $y_{1}$ is replaced by $y_{1}^{*}$. Thus, for $r=0, n$ even and sgn $K=(-)^{n / 2}$, the exact periodic solution of (1) is

$$
y=\frac{4|K|}{\pi \gamma^{n}} \sum_{s=1,3,5, \ldots} \frac{1}{s^{n+1}} \sin s(\gamma x+\phi),
$$

where $\phi$ and $\gamma$ are arbitrary.

We now derive (46) in closed form. If $r=0$, the right hand side of (1) will be constant in any interval in which $y$ does not change sign. By direct integration of (1) we obtain, during such interval,

$$
y(x)=\sum_{k=0}^{n} \frac{1}{k !} y^{(k)}\left(x_{0}\right)\left(x-x_{0}\right)^{k},
$$

where $x_{0}$ is arbitrary provided $x=x_{0}$ is within this interval. Suppose $x=x_{0}$ is chosen to be at the centre of the interval. Then, from (46), $\gamma x_{0}+\phi=\left(N+\frac{1}{2}\right) \pi$, where $N$ is integral. By differentiating (46) at $x=x_{0}$, we now obtain

$$
(-)^{N} y^{(k)}\left(x_{0}\right)=\left\{\begin{array}{l}
(-)^{k / 2} \frac{4|K|}{\pi \gamma^{n-k}} \sum_{:=1,3,5, \cdots} \frac{(-1)^{(s-1) / 2}}{s^{n+1-k}}, \text { if } k=0,2,4, \cdots n, \\
0, \quad \text { if } k \text { odd. }
\end{array}\right.
$$

Now, if $E_{n}$ is Euler's number of order $n\left(E_{0}=E_{1}=1, E_{2}=5, E_{3}=61, E_{4}=1385\right.$, 
$\cdots)$, it is known $[6,7]$ that

$$
\frac{E_{n}}{(2 n) !}=\frac{2^{2 n+2}}{\pi^{2 n+1}}\left[1-\frac{1}{3^{2 n+1}}+\frac{1}{5^{2 n+1}}-\frac{1}{7^{2 n+1}}+\cdots\right] .
$$

Applying (49) to (48) and substituting the resulting closed form of $y^{(k)}\left(x_{0}\right)$ into (47), we obtain as the exact periodic solution of (1) when $r=0, n$ is even and $\operatorname{sgn} K=(-)^{n / 2}$, $y= \pm \frac{K X^{n}}{n !}\left[\left(\frac{x-x_{0}}{X}\right)^{n}-{ }^{n} C_{2} E_{1}\left(\frac{x-x_{0}}{X}\right)^{n-2}\right.$

$$
\left.+{ }^{n} C_{4} E_{2}\left(\frac{x-x_{0}}{X}\right)^{n-4}-\cdots+(-)^{n / 2} E_{n / 2}\right], \quad X \geq\left|x-x_{0}\right|,
$$

where, for convenience, $\gamma$ has been replaced by the quarter period $X=\pi / 2 \gamma$, and ${ }^{n} C_{k}$ is the binomial coefficient $n ! /[(n-k) ! k !]$.

In (50), $x_{0}, X$ and the ambiguous sign are arbitrary. Solution (50) is valid for a half period. It is repeated by alternate positive and negative half cycles outside the range $X \geq\left|x-x_{0}\right|$. The maximum $A^{*}$ of $y$ occurs at $x=x_{0}$, and is, from (50),

$$
A^{*}=\frac{E_{n / 2}}{n !}|K| X^{n} \text {. }
$$

Alternatively, by chosing $x_{0}$ such that $\gamma x_{0}+\phi=N \pi$ ( $N$ integral), it can be shown simiarly that the exact solution of (1) when $r=0$, valid for a half period, is

$$
\begin{gathered}
y= \pm K(2 X)^{n}\left\{\frac{1}{n !}\left(\frac{x-x_{0}}{2 X}\right)^{n}-2\left[\frac{2^{2}-1}{2 !(n-1) !} B_{1}\left(\frac{x-x_{0}}{2 X}\right)^{n-1}-\frac{2^{4}-1}{4 !(n-3) !}\right.\right. \\
\left.\left.\cdot B_{2}\left(\frac{x-x_{0}}{2 X}\right)^{n-3}+\cdots-(-)^{n / 2} \frac{2^{n}-1}{n !} B_{n / 2}\left(\frac{x-x_{0}}{2 X}\right)\right]\right\}, \quad x_{0} \leq x \leq x_{0}+2 X,
\end{gathered}
$$

where the half period $2 X$ and $x_{0}$ are arbitrary. The $B_{n}$ are Bernoulli's numbers $\left(B_{1}=\frac{1}{6}\right.$, $\left.B_{2}=\frac{1}{30}, B_{3}=\frac{1}{42}, B_{4}=\frac{1}{30}, \cdots\right)$. The result $[6,7]$

$$
B_{n}=\frac{(2 n) ! 2}{\left(2^{2 n}-1\right) \pi^{2 n}}\left[1+\frac{1}{3^{2 n}}+\frac{1}{5^{2 n}}+\frac{1}{7^{2 n}}+\cdots\right]
$$

has been used in the derivation of (52).

6. Accuracy of converging and diverging oscillatory solutions. The accuracy of solutions (33) and (35) depends on the accuracy of the periodic approximate solution

$$
\eta_{1}=A_{m} \sin \left(\beta_{m} \theta+\phi\right)
$$

of (26), where $\beta_{m}$ is given by (30) and (32) as appropriate, and $A_{m}$ is given by (34).

Regarding $\eta_{1}$ as a first approximation to the true periodic solution of (26), we obtain an improved approximation $\eta_{1}^{*}$ by substituting $\eta_{1}$ into the right hand side of (26):

$$
\begin{aligned}
\left(\frac{d}{d \theta}+q\right)\left(\frac{d}{d \theta}+q-1\right) \cdots\left(\frac{d}{d \theta}+q-n+1\right) \eta_{1}^{*} & \\
& =K A_{m}^{n} c_{r} \sum_{r=1,3,5, \ldots} h_{r, s} \sin s\left(\beta_{m} \theta+\phi\right),
\end{aligned}
$$

where $c_{r}$ is given by (18) and $h_{r, \text { by }}(42)$. 
Integrating (54) yields

$$
\eta_{1}^{*}=\operatorname{Im} \sum_{s=1,3,5, \ldots}\left[A_{m} \frac{\left(j \beta_{m}+q\right)\left(j \beta_{m}+q-1\right) \cdots\left(j \beta_{m}+q-n+1\right)}{\left(j s \beta_{m}+q\right)\left(j s \beta_{m}+q-1\right) \cdots\left(j s \beta_{m}+q-n+1\right)} h_{r, s} e^{j s\left(\beta_{m} \theta+\phi\right)}\right],
$$

using (28).

If $e$ is a measure of harmonic content, defined as before, we have from (55)

$$
e^{2}=\sum_{s=3,5,7, \ldots} \frac{\left(\beta_{m}^{2}+q^{2}\right)\left(\beta_{m}^{2}+(q-1)^{2}\right) \cdots\left(\beta_{m}^{2}+(q-n+1)^{2}\right)}{\left(s^{2} \beta_{m}^{2}+q^{2}\right)\left(s^{2} \beta_{m}^{2}+(q-1)^{2}\right) \cdots\left(s^{2} \beta_{m}^{2}+(q-n+1)^{2}\right)} h_{r, s}^{2} .
$$

If $0 \leq r \leq 3$, and the smallest $\beta_{m}$ is selected in each case, it is found that $e<0.02$ if $n \leq 8$ and $e<0.04$ if $n \leq 16$. If alternative values of $\beta_{m}$ are selected, $e$ is reduced in each case. These figures suggest that amplitude $A_{m}$ and frequency $\beta_{m}$ are determined by (34) and (30) or (32) within at least $2 \%$ or $4 \%$ when $n \leq 8$ or $n \leq 16$ respectively and $0 \leq r \leq 3$.

Using the changes of variable (24) and (25) we obtain from (55), an improved approximation for the solution of (1), which is most conveniently written in the form

$$
\begin{aligned}
y=\operatorname{Im} \sum_{s=1,3,5, \ldots} A_{m} \frac{\left(j \beta_{m}+q\right)\left(j \beta_{m}+q-1\right) \cdots\left(j \beta_{m}+q-n+1\right)}{\left(j s \beta_{m}+q\right)\left(j s \beta_{m}+q-1\right) \cdots\left(j s \beta_{m}+q-n+1\right)} & \\
& \times h_{r, s}\left(x-x_{0}\right)^{q+i s \beta_{m}} e^{i s \phi}, \quad x>x_{0} .
\end{aligned}
$$

The first term of the sum in (57) is identical with the approximate solution (33). Expression (57) should be used if derivatives of $y$ are required to the same accuracy as (33).

The improved approximation of solution (35), valid for $x_{0}>x$, is identical to (57) with $x-x_{0}$ replaced by $x_{0}-x$.

Exact converging and diverging oscillatory solutions when $r=0$. When $r=0$, the improved approximate solution (55) of (26) can be written

$$
\eta_{1}^{*}(\theta)=\frac{4 K}{\pi} \operatorname{Im} \sum_{s=1,3,5, \ldots} \frac{e^{i s\left(\beta_{m} \theta+\phi\right)}}{s\left(1+j s \beta_{m}\right)\left(2+j s \beta_{m}\right) \cdots\left(n+j s \beta_{m}\right)} .
$$

On substituting (58) into (26) with $r=0$, it is evident that (58) is an exact solution of (26) if $\beta_{m}\left(\beta_{m}>0\right)$ is chosen so that $\eta_{1}^{*}=0$ and $d \eta_{1}^{*} / d \theta>0$ when $\beta_{m} \theta+\phi=2 N \pi$ ( $N$ integral). Let the modified values of $\beta_{m}$ which satisfy this condition be denoted by $\beta_{m}^{*}$.

From (24) and (25) the corresponding exact solution of (1) is

$$
y(x)=\frac{4 K}{\pi} \operatorname{Im} \sum_{s=1,3,5, \ldots} \frac{\left(x-x_{n}\right)^{n+i s \beta_{m}{ }^{*}} e^{j s \phi}}{s\left(1+j s \beta_{m}^{*}\right)\left(2+j s \beta_{m}^{*}\right) \cdots\left(n+j s \beta_{m}^{*}\right)} .
$$

Also, from (24) and (25) we have

$$
\eta_{1}^{*}=0 \text { and } \operatorname{sgn} d \eta_{1}^{*} / d \theta=\operatorname{sgn} y^{(1)}(x) \text { when } y=0 .
$$

Hence, if $x=x_{0}+X$ is a zero of $y, \beta_{m}^{*}$ can be obtained from the conditions

$$
y\left(x_{0}+X\right)=0 \text { and } y^{(1)}\left(x_{0}+X\right)>0 \text { when } 2 N \pi=\beta_{m}^{*} \bar{\theta}+\phi,
$$

where $X=e^{\bar{\theta}}$, a constant. Now, from (61)

$$
X^{i s \beta_{m}^{*}} e^{i s \phi}=1 .
$$


Hence, by differentiating (59) at $x=x_{0}+X$, we obtain

$$
\begin{array}{r}
y^{(k)}\left(x_{0}+X\right)=\frac{4 K}{\pi} \operatorname{Im} \sum_{s=1,3,5, \ldots} \frac{X^{n-k}}{s\left(1+j s \beta_{m}^{*}\right)\left(2+j s \beta_{m}^{*}\right) \cdots\left(n-k+j s \beta_{m}^{*}\right)} \\
(k=0,1,2, \cdots n-1),
\end{array}
$$

where $\beta_{m}^{*}\left(\beta_{m}^{*}>0\right)$ is such that, in (63),

$$
y^{(0)}\left(x_{0}+X\right)=0 \text { and } y^{(1)}\left(x_{0}+X\right)>0 .
$$

Expressions (63) are now converted into closed form. If the right hand side is expanded into partial fractions we obtain

$$
y^{(k)}\left(x_{0}+X\right)=\frac{4 K}{\pi} \sum_{s=1,3,5, \ldots} \sum_{h=1}^{n-k}(-)^{h} \frac{X^{n-k}}{h !(n-k-h) !} \frac{h \beta_{m}^{*}}{\left(h^{2}+s^{2} \beta_{m}^{* 2}\right)} .
$$

The series expansion

$$
\tanh u=8 u\left[\frac{1}{\pi^{2}+4 u^{2}}+\frac{1}{9 \pi^{2}+4 u^{2}}+\frac{1}{25 \pi^{2}+4 u^{2}}+\cdots\right]
$$

can be derived by replacing $u$ by $j u$ in the corresponding better known expansion $[6,7]$ for $\tan u$. With $u=\frac{1}{2} \pi h / \beta_{m}^{*}$, expansion (66) may be applied directly to (65) with the order of summation reversed, to yield

$$
y^{(k)}\left(x_{0}+X\right)=\frac{K X^{n-k}}{(n-k) !} F_{n-k}\left(\rho_{m}\right) \quad(k=0,1,2, \cdots n-1) .
$$

where

$$
\rho_{m}=e^{\pi / \beta_{m}^{*}}
$$

and

$$
F_{i}(\rho)=\sum_{h=1}^{i}(-)^{h}{ }^{i} C_{h} \frac{\rho^{h}-1}{\rho^{h}+1} \quad(i=1,2, \cdots) .
$$

Since $\sum_{h=0}^{i}(-)^{h}{ }^{i} C_{h}=0$, an alternative form of (69) is

$$
F_{i}(\rho)=-2 \sum_{h=0}^{i}(-)^{h}{ }^{i} C_{h} \frac{1}{1+\rho^{h}} \quad(i=1,2, \cdots) .
$$

A further alternative form of $F_{i}(\rho)$ is given in the Appendix.

Conditions (64) applied to (67) show that $\rho_{m}$ is any root of

$$
F_{n}(\rho)=0
$$

such that

$$
K F_{n-1}\left(\rho_{m}\right)>0 \text { and } \rho_{m}>1 .
$$

The condition $\rho_{m}>1$ is necessary and sufficient to ensure $\beta_{m}^{*}>0$, from (68).

Now, if (1) is integrated during an interval in which $y$ is positive, we have in terms of initial conditions at $x=x_{0}+X$

$$
y=\frac{K}{n !}\left(x-x_{0}-X\right)^{n}+\sum_{k=1}^{n-1} \frac{1}{k !} y^{(k)}\left(x_{0}+X\right)\left(x-x_{0}-X\right)^{k}
$$


On substituting from (67), we obtain finally, as an exact solution of (1) when $r=0$,

$$
\begin{aligned}
y= \pm & \frac{K X^{n}}{n !}\left[\left(\frac{x-x_{0}-X}{X}\right)^{n}+{ }^{n} C_{1} F_{1}\left(\rho_{m}\right)\left(\frac{x-x_{0}-X}{X}\right)^{n-1}+{ }^{n} C_{2} F_{2}\left(\rho_{m}\right)\left(\frac{x-x_{0}-X}{X}\right)^{n-2}\right. \\
& \left.+\cdots+{ }^{n} C_{n-1} F_{n-1}\left(\rho_{m}\right)\left(\frac{x-x_{0}-X}{X}\right)\right], \quad x_{0}+X \leq x \leq x_{0}+\rho_{m} X,
\end{aligned}
$$

where $X$ and $x_{0}$ are arbitrary. The following notes explain certain features of solution (74).

(a) In (74) the ambiguous sign has been introduced to allow for an interval in which $y$ is negative.

(b) The $F_{i}$ are defined by (69) or (70); $\rho_{m}$ must be determined from (71) with (72). For $n \leq 8$ all the required values of $\rho_{m}$ and $F_{i}\left(\rho_{m}\right)(i<n)$ are given in Table 3.

(c) From (68), (58) and (25), zeros of $y$ occur at $x=x_{0}+X, x_{0}+\rho_{m} X, x_{0}+\rho_{m}^{2} X, \cdots$. Hence $\rho_{m}$ is the ratio of the length of an interval between successive zeros to the length of the previous interval between successive zeros.

(d) A corollary of note (c) is that during the $r$-th interval following that for which (74) is valid, $y$ is given by the right hand side of (74) multiplied by $(-1)^{|r|}$ and with $X$ replaced by $\rho_{m}^{r} X$. This defines the solution completely for $x>x_{0}$ ( $r$ may be negative).

The above derivation applies for diverging oscillatory solutions of (1) when $r=0$. The corresponding result for converging oscillatory solutions is obtained by replacing $x-x_{0}$ by $x_{0}-x$ in (74) and $K$ by $(-)^{n} K$ in (74) and (72). The notes following (74) also apply, provided $x-x_{0}$ is replaced by $x_{0}-x$ and the words "following" and "previous" are interchanged. It will be noted, from (71) and (72), that the $\rho_{m}$ are the same for converging and diverging oscillatory solutions if $n$ is even, but are different if $n$ is odd.

The accuracy of approximate solutions (33) and (35) when $r=0$ is illustrated by the remarkable agreement between values of $e^{\pi / \beta_{m}}$ and $e^{\pi / \beta_{m} *}\left(=\rho_{m}\right)$, as shown in Table 3 .

7. Superposition of solutions if $r<1$. The solutions (15) and (33) are not defined for $x<x_{0}$, and the solutions (11) and (35) are not defined for $x>x_{0}$. For all these solutions it may be verified that, if $r<1, y$ and its first $n$-1 derivatives become zero as $x$ approaches $x_{0}$. Now, $y=0$ is also a solution of (1). Hence, if $r<1$, the range of solutions (15) and (33) may be extended by taking $y=0$ for $x \leq x_{0}$, and the range of solutions (11) and (35) may be extended by taking $y=0$ for $x \geq x_{0}$. Furthermore, the sum of a converging solution and a diverging solution, each so defined for all $x$, will also satisfy (1) provided they are not both non-zero at any $x$.

The following is a simple example in which solutions may be superimposed. A light uniform beam of length $L$ is simply supported at each end. The beam is loaded at each end by a tensile force $P$ acting along the line joining the supports. The material of the beam is such that the curvature of the beam is $k M^{1 / 3}$ (independent of the local tension), where $M$ is the local bending moment. Hogging moments $M_{1}$ and $M_{2}$ are applied at each support.

It is easy to show that $M$ satisfies

$$
\frac{d^{2} M}{d x^{2}}=P k M^{1 / 3},
$$

with boundary conditions $M=M_{1}$ at $x=0$ and $M=M_{2}$ at $x=L$. It can be verified directly that a solution of (75) which satisfies the boundary conditions is 


$$
M= \begin{cases}\left(M_{1}^{1 / 3}-\alpha x\right)^{3}, & \text { if } \alpha x<M_{1}^{1 / 3}, \\ 0, & \text { if } \quad M_{1}^{1 / 3} \leq \alpha x \leq \alpha L-M_{2}^{1 / 3}, \\ {\left[\alpha(x-L)+M_{2}^{1 / 3}\right]^{3},} & \text { if } \quad \alpha L-M_{2}^{1 / 3}<\alpha x,\end{cases}
$$

where $\alpha=\sqrt{ }(k P / 6)$, provided

$$
\alpha L>M_{1}^{1 / 3}+M_{2}^{1 / 3} \text {. }
$$

This solution is made up from (11) and (15) in non-overlapping ranges of $x$, and connected by the solution $M=0$. If the ranges overlap, i.e. the beam is too short to satisfy (76), then a different form of solution must be expected.

8. Summary of results. The following special solutions of $d^{n} y / d x^{n}=K|y|^{r} \operatorname{sgn} y$ have been derived. Exact solutions (11) and (15) are for converging and diverging monotonic modes. Approximate solution (22) is for a periodic mode, with (43) as a refined approximation. If $r=0$, the refined approximation (43) for a periodic solution is exact, and is given in closed form by (50) or (52). Approximate solutions (33) and (35) are for diverging and converging oscillatory modes; a refinement of these solutions is given by (57) and a modified form of (57). If $r=0$, diverging oscillatory modes are given exactly by (74) and converging oscillatory modes by a modified form of (74). For $n \leq 8$ and $0 \leq r \leq 3$, all the approximate solutions are expected to be accurate within about $2 \%$.

The number of distinct solutions of each type (converging monotonic, diverging monotonic, periodic, converging oscillatory, diverging oscillatory) depends on $n$ and the sign of $K$, but not on $r$, and is the same as in the linear case $r=1$ as shown in Table

TABLE 3

\begin{tabular}{|c|c|c|c|c|c|c|}
\hline$n$ & 3 & 4 & 5 & 5 & 6 & 6 \\
\hline$e^{\tau / \beta_{m}}$ & 2.57855 & 4.07538 & 5.55607 & 1.41579 & 7.03823 & 1.79647 \\
\hline$\rho_{m}$ & 2.61803 & 4.13016 & 5.60796 & 1.41635 & 7.07175 & 1.73691 \\
\hline $10^{5} F_{1}\left(\rho_{m}\right)$ & -44721.4 & -61014.9 & -69733.5 & -17230.7 & -75222.2 & -26924.8 \\
\hline $10^{5} F_{2}\left(\rho_{m}\right)$ & -14907.1 & -33105.0 & -45630.4 & -993.648 & -54365.3 & -3639.93 \\
\hline $10^{5} F_{3}\left(\rho_{m}\right)$ & 0 & -13471.4 & -26563.2 & 776.941 & -36865.2 & 1906.02 \\
\hline $10^{5} F_{4}\left(\rho_{m}\right)$ & - & 0 & -11606.3 & 340.158 & -22238.1 & 1965.11 \\
\hline $10^{5} F_{5}\left(\rho_{m}\right)$ & - & - & 0 & 0 & -10068.5 & 889.033 \\
\hline$n$ & 7 & 7 & 7 & 8 & 8 & 8 \\
\hline$e^{\pi / \beta_{m}}$ & 8.52651 & 2.00816 & 1.19903 & 10.02203 & 2.24972 & 1.35085 \\
\hline$\rho_{m}$ & 8.52851 & 2.00832 & 1.19906 & 9.98125 & 2.24960 & 1.35086 \\
\hline $10^{5} F_{1}\left(\rho_{m}\right)$ & -79010.3 & -33517.8 & -9051.95 & -81787.1 & -38453.9 & -14924.8 \\
\hline $10^{5} F_{2}\left(\rho_{m}\right)$ & -60733.1 & -6770.44 & -147.134 & -65561.8 & -9907.40 & -650.411 \\
\hline $10^{5} F_{3}\left(\rho_{m}\right)$ & -44846.4 & 2219.35 & 137.720 & -51123.2 & 1788.80 & 541.768 \\
\hline $10^{5} F_{4}\left(\rho_{m}\right)$ & -31066.0 & 3846.77 & 17.5750 & -38290.4 & 5268.05 & 180.869 \\
\hline $10^{5} F_{5}\left(\rho_{m}\right)$ & -19141.4 & 2864.68 & -5.93640 & -26900.8 & 5060.10 & -18.9386 \\
\hline $10^{5} F_{6}\left(\rho_{m}\right)$ & -8851.13 & 1320.97 & -2.73489 & -16808.1 & 3471.97 & -45.8812 \\
\hline $10^{5} F_{7}\left(\rho_{m}\right)$ & 0 & 0 & 0 & -7800.69 & 1621.10 & -21.4000 \\
\hline
\end{tabular}

All roots $\rho_{m}\left(\rho_{m}>1\right)$ of $F_{n}(\rho)=0(n \leq 8)$ and the corresponding values of $F_{i}\left(\rho_{m}\right)(i<n)$ 
1. We have not attempted to prove rigorously that exact solutions corresponding to the approximate solutions exist; that such exact solutions do exist is known [5] for $n=2, r>0$, and has been verified here for $n \leq 8, r=0$.

\section{Appendix}

An alternative form of $F_{i}(\rho)$, defined by $(70)$, is

$$
F_{i}(\rho)=\frac{i !(1-\rho)^{i}}{(1+\rho)\left(1+\rho^{2}\right) \cdots\left(1+\rho^{i}\right)} \Delta_{i}(\rho),
$$

where $\Delta_{i}(\rho)$ is the reciprocal polynomial of $\frac{1}{2} i(i-1)$-th degree, defined by

$$
\Delta_{i}(\rho)=\left|\begin{array}{cccccc}
\frac{1}{1 !} & \frac{1}{2 !} & \frac{1}{3 !} & \frac{1}{4 !} & \cdots & \frac{1}{i !} \\
1+\rho^{i-1} & \frac{1}{1 !} & \frac{1}{2 !} & \frac{1}{3 !} & \cdots & \frac{1}{(i-1) !} \\
0 & 1+\rho^{i-2} & \frac{1}{1 !} & \frac{1}{2 !} & \cdots & \frac{1}{(i-2) !} \\
0 & 0 & 1+\rho^{i-3} & \frac{1}{1 !} & \cdots & \frac{1}{(i-3) !} \\
\vdots & \vdots & \vdots & \vdots & & \vdots \\
0 & 0 & 0 & 0 & \cdots & \frac{1}{1 !}
\end{array}\right|
$$

On expanding $\Delta_{i}$ in terms of the elements of its first row, we obtain a recurrence relation from which $\Delta_{1}, \Delta_{2}, \cdots$ can be successively calculated:

$$
\begin{aligned}
\Delta_{n}=\frac{1}{1 !} \Delta_{n-1}-\frac{1}{2 !}\left(1+\rho^{n-1}\right) \Delta_{n-2} & +\frac{1}{3 !}\left(1+\rho^{n-1}\right)\left(1+\rho^{n-2}\right) \Delta_{n-3}-\cdots \\
& +(-)^{n-1} \frac{1}{n !}\left(1+\rho^{n-1}\right)\left(1+\rho^{n-2}\right) \cdots(1+\rho) .
\end{aligned}
$$

Identity (77) can be proved by induction, using (79). Also $\Delta_{i}(\rho)$ is clearly a polynomial of $\frac{1}{2} i(i-1)$-th degree, by inspection of $(78)$. It is reciprocal because $F_{i}(\rho)=$ $-F_{i}(1 / \rho)$, from $(69)$.

An alternative derivation of exact solutions of (1) when $r=0$, in which the polynomials $\Delta_{i}$ arise naturally, is given in [1] for the case $n=6$.

\section{REFERENCES}

1. P. E. W. Grensted and A. T. Fuller, Minimization of integral-square-error for non-linear control systems of third and higher order, International J. Contr., II (1965) p. 33

2. W. J. Cunningham, Introduction to nonlinear analysis, McGraw-Hill, New York (1958)

3. H. D. Greif, Describing function method of servomechanism analysis applied to most commonly encountered nonlinearities, Trans. Amer. Instn. Elect. Engrs., 72, part II (Applications and Industry), (1953) p. 243

4. H. Kauderer, Nichtlineare Mechanik, Springer-Verlag, Berlin, (1958) p. 209

5. R. M. Rosenberg, The Ateb(h)-functions and their properties, Q. Appl. Math., 21 (1963) p. 37

6. N. R. C. Dockeray, Elementary treatise on pure mathematics, Bell, London, (1934) chapter XV

7. B. O. Pierce and R. M. Foster, A short table of integrals, Fourth Ed., Ginn, Boston (1956) 